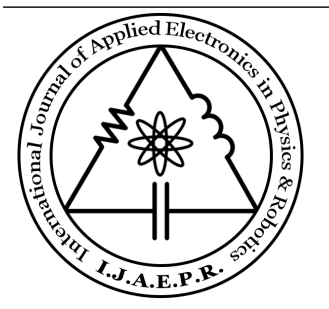

Research Article

\title{
Stress Analysis and SERR Calculation in Fiber/Matrix Interfacial Crack
}

\author{
Ahamd Ghanbari $^{1,2, *}$, Arash Rahmani ${ }^{2}$, Ramin Soleimani ${ }^{3}$, Farshid Tabatabaie $^{3}$ \\ (1) School of Engineering Emerging Technologies, Mechatronic Laboratory. University of Tabriz, Tabriz, Iran. \\ (2) Department of Mechanical Engineering, University of Tabriz, Tabriz, Iran. \\ (3) Department of Engineering, Ajabshir Branch, Islamic Azad University, Ajabshir, Iran.
}

\section{Article history:}

Received 10 April 2013

Reviewed 18 April 2013

Revised 21 April 2013

Accepted 23 April 2013

Published 6 July 2013

\begin{abstract}
Stress analysis method was used to establish a theoretical model to find the energy release rate for initiation of an interfacial crack and progressive debonding with friction at debonded interface. For this propose, using stress equilibrium equations, boundary and continuity conditions and minimum complementary energy principle, we defined an expression for energy release rate, $G$, for a single fibre embedded in a concentric cylindrical matrix, to explore the fibre/matrix interfacial fracture properties. We determine the critical crack length by interfacial debonding criterion. Also, Numerical calculation results for fibre-reinforced composite, SiC/LAS, were compared with experimental data witch obtained by other methods.
\end{abstract}

Keywords: energy release rate, fiber-reinforced composite, interfacial crack, Stress analysis.

\section{Introduction}

For both theoretical analysis and experimental studies to develop a successful fiber-reinforced composite, the properties of the fiber/matrix interface have been identified as a key factor. The most important of these properties is the constraint between the fiber and matrix that is related to slipping and debonding, which is associated with the work of fracture for composite failure. Therefore, many researches have used single fiber composites model to study and explore the initiation and progressing of interfacial fiber/matrix debonding. By testing fiber pullout, Hampe et al. pointed out that a debonded interface may appear before the interfacial shear stress reaches the shear strength, which shows that the shear strengthbased criterion is invalid to some extent ${ }^{[1]}$. Honda and Kagawa showed that, according to the energy-based interfacial debonding criterion, the interface crack grows when the energy release rate $G$ exceeds the interfacial debonding toughness ${ }^{[2]}$. By applying the shear-lag models and the Lamé method respectively, Hsueh and Ochiai et al. obtained solutions for the energy release rate and the bridging law ${ }^{[3,4]}$. However, they neglected the shear stress and strain energy in the fiber, the interfacial radial stress, the variation of axial stress in the matrix with radial positions, and the Poisson's effect. When the axial stress in the matrix is substituted by an equivalent axial stress concentrating on an effective radius, Chiang further derived an expression for the energy release rate including the axial strain energy in the fiber,

\footnotetext{
${ }^{*}$ Corresponding author: A. Ghanbari

ত: +98 (0)914 1160796

$凶$ : a-ghanbari@tabrizu.ac.ir
}

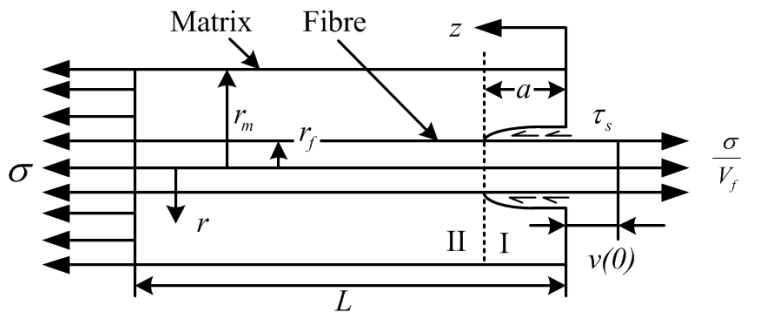

Fig. 1. A composite cylindrical model of length L having an interracial crack of length a

and the axial and shear strain energy in the matrix ${ }^{[5]}$. Rauchs and Withers obtained numerical solutions of the energy release rate by using the finite element method ${ }^{[6]}$. However, oversimplifications resulted in serious errors. Damage growth by debonding in a single fibre metal matrix composite is investigated by Papakaliatakis and Karalekas ${ }^{[7]}$. They Elastoplasticity and strain energy density criterion for this propose. Kushch et al. are applied numerical simulation of progressive debonding in fiber reinforced composite under transverse loading ${ }^{[8]}$. Johnson et al. studied the role of matrix cracks and fibre/matrix debonding on the stress transfer between fibre and matrix in a single fibre fragmentation test ${ }^{[9]}$.

In this paper we extract an expression for strain energy release rate (SERR) for a crack propagation analysis by using stress equilibrium equations, boundary and continuity conditions and minimum complementary energy principle. For this propose, as shown in Fig. 1 a single fiber embedded in a concentric cylindrical matrix in a cylindrical coordinate $(r, z, \theta)$ is considered. In Fig. 1 a specimen with an interfacial crack with a crack length of 
$a$ is divided into two regions ${ }^{[10]}$ :

1. Region $I$ is the region within the interfacial crack.

2. Region II is the region with an intact region.

Our first step is to find stresses in each region. After that, we should calculate total strain energies in the specimen. Then, according to strain energy release rate definition we extract an expression for SERR.

\section{Stress Analysis}

\subsection{Region I}

Consider the equilibrium of the axial force acting on element of length in the fiber of region I, leads to the following equation.

$$
d \sigma_{f} \times\left(\pi r_{f}^{2}\right)=-\tau_{s} \times\left(\pi r_{f}\right) \cdot d z \Rightarrow \frac{d \sigma_{f}}{d z}=-\frac{2}{r_{f}} \tau_{s}
$$

Considering the boundary conditions as bellow.

$$
\begin{gathered}
\sigma(z=L)=\left(E_{f} / E\right) \sigma \\
\sigma_{m}(z=L)=\left(E_{m} / E\right) \sigma \\
\sigma_{f}(z=0)=\left(\sigma / V_{f}\right) \\
\sigma_{m}(z=0)=0
\end{gathered}
$$

and total axial stresses satisfaction

$$
V_{f} \sigma_{f}+V_{m} \sigma_{m}=\sigma
$$

Solving (1) and using (2) to (6), the fiber and matrix stresses in the cracked iterfacial length, region $\mathrm{I},(0 \leq z \leq$ a) become

$$
\begin{gathered}
\sigma_{f, I}(z)=\left(\frac{\sigma}{V_{f}}\right)-\left(\frac{2 \tau_{s}}{r_{f}}\right) z \\
\sigma_{m, I}(z)=\left(\frac{V_{f}}{V_{m}}\right)-\left(\frac{2 \tau_{s}}{r_{f}}\right) z
\end{gathered}
$$

where $a$ denote the crack length, $V_{m}, V_{f}$ show matrix and fiber volume fraction and $\tau_{s}, \sigma_{m}, \sigma_{f}$ and $\sigma$ are interfacial shear stress, matrix and fiber and far field axial stresses.

In the cylindrical coordinate, the equilibrium equations for a $3 \mathrm{D}$ axi-symmetric problem are given by

$$
\begin{gathered}
\frac{\partial \sigma_{r}}{\partial r}+\frac{\partial \tau_{r z}}{\partial z}+\frac{\sigma_{r}-\sigma_{\theta}}{r}=0 \\
\frac{\partial \sigma_{z}}{\partial z}+\frac{\partial \tau_{r z}}{\partial r}+\frac{\tau_{r z}}{r}=0
\end{gathered}
$$

Solving (9) and (10) and using boundary conditions as below.

$$
\begin{gathered}
\tau_{f}\left(r=r_{f}, z\right)=\tau_{m}\left(r=r_{f}, z\right) \\
=\tau_{s} \\
\tau_{m}\left(r=r_{m}, z\right)=0
\end{gathered}
$$

Shear stresses in region I are calculated.

$$
\begin{gathered}
\tau_{f, I}(r, z)=\frac{r}{r_{f}} \tau_{s} \\
\tau_{m, I}(r, z)=\frac{V_{f}\left(r_{m}^{2}-r^{2}\right)}{V_{m} r_{f} r} \tau_{s}
\end{gathered}
$$

\subsection{Region II}

For calculation the stresses in this region we introduce stress function as

$$
F_{k}=H_{k}(z) I_{k}(r)
$$

where $k=f, m$ represent the fiber and matrix, respectively. The stress solutions satisfying (9) and (10) at region II are expressed as

$$
\begin{aligned}
& \sigma_{k}(z)=\frac{\partial^{2} F_{k}}{\partial r^{2}}+\frac{1}{r} \frac{\partial F_{k}}{\partial r} \\
&=\left(\frac{\partial^{2} I_{k}(r)}{\partial r^{2}}+\frac{1}{r} \frac{\partial I_{k}(r)}{\partial r}\right) H_{k} \\
& \tau_{k}(r, z)=\frac{\partial^{2} F_{k}}{\partial r \partial z} \\
&=-\frac{\partial I_{k}}{\partial r} \cdot \frac{\partial H_{k}}{\partial z} \\
& \sigma_{k}(\theta)=\sigma_{k}(r) \\
&= \frac{\partial^{2} F_{k}}{\partial z^{2}} \\
&=I_{k}(r) \cdot \frac{\partial^{2} H_{k}}{\partial z^{2}}
\end{aligned}
$$

Therefore, using stress boundary conditions as below.

$$
\begin{aligned}
& \left\{\begin{array}{l}
\sigma_{f, I I}(z=a)=\frac{\sigma}{V_{f}}-\frac{2 \tau_{s} a}{r_{f}} \\
\sigma_{m, I I}(z=a)=\frac{2 V_{f} \tau_{s} a}{V_{m} r_{f}}
\end{array}\right. \\
& \left\{\begin{array}{l}
\sigma_{f, I I}(z=L)=\frac{E_{f}}{E} \sigma \\
\sigma_{m, I I}(z=L)=\frac{E_{m}}{E} \sigma
\end{array}\right. \\
& \sigma_{f}\left(r=r_{f}\right)=\sigma_{m}\left(r=r_{f}\right) \\
& \tau_{f}\left(r=r_{f}, z\right)=\tau_{m}\left(r_{f}, z\right) \\
& =\tau_{i}(z) \\
& \sigma_{m}\left(r, r_{m}\right)=\tau_{m}\left(r=r_{m}, z\right) \\
& =0
\end{aligned}
$$

where $r_{f}, r_{m}$ and $\tau_{i}(z)$ denote fiber and matrix radius and interfacial shear stress in region II, respectively.

By solving (16) and substituting (19) the functions $I_{f}$, $I_{m}$ and $H_{m}$ are expressed as

$$
\begin{gathered}
I_{f}=\frac{1}{4}\left(\frac{\sigma}{V_{f}}-\frac{2 \tau_{s} a}{r_{f}}\right) r^{2}+A_{f} \\
I_{m}(r)=A_{m}\left(r^{2}\right)+B_{m} \operatorname{Ln}(r)+C_{m} \\
H_{m}=\frac{\sigma}{4 V_{m} A_{m}}\left(1+\frac{2 \tau_{s} a}{r_{f}} H_{f}\right)
\end{gathered}
$$

where $A_{f}, A_{m}, B_{m}$ and $C_{m}$ are constant coefficients which by using (20) to (23) are calculated as

$$
\begin{aligned}
A_{f} & =\frac{1}{4}\left(\frac{\sigma}{V_{f}}-\frac{2 \tau_{s} a}{r_{f}}\right) \\
& \times\left(\frac{V_{f}}{V_{m}}\left(r_{f}^{2}-r_{m}^{2}-2 r_{m}^{2} \operatorname{Ln} \frac{r_{m}}{r_{f}}\right)+r_{f}^{2}\right)
\end{aligned}
$$




$$
\begin{gathered}
A_{m}=-\frac{V_{f}}{4 V_{m}}\left(\frac{\sigma}{V_{f}}-\frac{2 \tau_{s} a}{r_{f}}\right) \\
B_{m}=-\left(\frac{V_{f}}{V_{m}}\right)\left(\frac{r_{m}^{2}}{2 L n r_{m}}\right)\left(\frac{\sigma}{V_{f}}-\frac{2 \tau_{s} a}{r_{f}}\right) \\
C_{m}=\left(\frac{V_{f} r_{m}^{2}}{4 V_{m}}\right)\left(\frac{\sigma}{V_{f}}-\frac{2 \tau_{s} a}{r_{f}}\right)
\end{gathered}
$$

By substituting (27) to (30) into (24) and (25) and using (16) to (18), the stresses in region II are written as

$$
\begin{gathered}
\sigma_{f, I I}(z)=\left(\frac{V_{m} E_{m} \sigma}{V_{f} E}-\frac{2 \tau_{s} a}{r_{f}}\right) \lambda(z)+\frac{E_{f}}{E} \sigma \\
\sigma_{m, I I}(z)=\left(\frac{V_{f} \tau_{s} a}{V_{m} r_{f}}-\frac{E_{m} \sigma}{E}\right) \lambda(z)+\frac{E_{m}}{E} \sigma \\
\tau_{i}(z)=\frac{\psi}{2}\left(\frac{V_{m} E_{m} \sigma}{V_{f} E}-\frac{2 \tau_{s} a}{r_{f}}\right) \lambda(z) \\
\tau_{f, I I}(r, z)=\frac{r \psi}{2 r_{f}}\left(\frac{V_{m} E_{m} \sigma}{V_{f} E}-\frac{2 \tau_{s} a}{r_{f}}\right) \lambda(z) \\
\tau_{m, I I}(r, z)=\frac{V_{f}\left(r_{m}^{2}-r^{2}\right) \psi}{2 V_{m} r_{f} r} \\
\times\left(\frac{V_{m} E_{m} \sigma}{V_{f} E}-\frac{2 \tau_{s} a}{r_{f}}\right) \lambda(z)
\end{gathered}
$$

$$
\begin{aligned}
\sigma_{f, I I}(r) & =\sigma_{f, I I}(\theta) \\
& =\frac{\left(r^{2}-r_{f}^{2}-\frac{V_{f}\left(r_{f}^{2}-r_{m}^{2}\left(1-2 L n \frac{r_{f}}{r_{m}}\right)\right)}{V_{m}}\right) \psi^{2}}{4 r_{f}^{2}} \\
& \times\left(\left(\frac{V_{m} E_{m} \sigma}{V_{f} E}-\frac{2 \tau_{s} a}{r_{f}}\right) \lambda(z)\right)
\end{aligned}
$$$$
\sigma_{f, I I}(r)=\sigma_{f, I I}(\theta)
$$$$
=\frac{\left(r^{2}-r_{m}^{2}\left(1-2 \operatorname{Ln} \frac{r_{f}}{r_{m}}\right)\right) \psi^{2}}{4 r_{f}^{2}}
$$$$
\times\left(\left(\frac{2 V_{f} \tau_{s} a}{V_{m} r_{f}}-\frac{E_{m} \sigma}{E}\right) \lambda(z)\right)
$$

where

$$
\lambda(z)=e^{-\psi(z-a) / r_{f}}
$$

and

$$
\psi=\frac{E}{E_{f} V_{m}\left(1-v_{m}\right) \phi}
$$

And $\phi$ is a non-dimensional parameter, given by Budiansky and $\mathrm{Cui}^{[11]}$ as

$$
\phi=-\frac{1}{2 V_{m}^{2}}\left(2 \operatorname{Ln} V_{f}+V_{m}\left(3-V_{f}\right)\right)
$$

In above equations $E, E_{m}$ and $E_{f}$ are Young's modulus of composite, matrix and fiber, respectively, and $v_{m}$ denotes the Poisson's ratio for matrix.

\section{Strain Energy Release Rate}

The total strain energy in the specimen is

$$
\Pi=U_{I}+U_{I I}+U_{W}
$$

where $U_{I}$ and $U_{I I}$ denote the total strain energy in region I and II, respectively. $U_{W}$ is the work done by external forces. Therefore, according to crack propagation concept and Minimum complementary energy principle, the total energy release rate associated with growth of crack in Fig. 1 is

$$
\begin{aligned}
G & =\frac{\partial \Pi}{\partial A} \\
& =\frac{-1}{2 \pi r_{f}} \cdot \frac{\partial \Pi}{\partial a} \\
\Rightarrow G & =\frac{-1}{2 \pi r_{f}}\left(\frac{\partial U_{I}}{\partial a}+\frac{\partial U_{I I}}{\partial a}+\frac{\partial U_{W}}{\partial a}\right)
\end{aligned}
$$

Then, we should calculate $U_{I}, U_{I I}$ and $U_{W}$ separately.

$$
U_{i}=U_{f, i}+U_{m, i} \quad i=I, I I
$$

In (43), $U_{f, i}$ and $U_{m, i}$ are total strain energy for fiber and matrix respectively, in region I and II with respect to suffix $i$ and according to strain energy definition is expressed as

$$
\begin{aligned}
U_{f, i} & =\int_{0}^{2 \pi} \int_{0}^{r_{f}} \int_{x}^{y} \frac{\sigma_{f, i}(z) \varepsilon_{f}(z)}{2} \\
& +\frac{\sigma_{f, i}(r) \varepsilon_{f}(r)}{2}+\frac{\sigma_{f, i}(\theta) \varepsilon_{f}(\theta)}{2} \\
& +\tau_{f, i}(r, z) \varepsilon_{f}(r, z) r d z d r d \theta \\
U_{m, i} & =\int_{0}^{2 \pi} \int_{0}^{r_{m}} \int_{x}^{y} \frac{\sigma_{m, i}(z) \varepsilon_{m}(z)}{2} \\
& +\frac{\sigma_{m, i}(r) \varepsilon_{m}(r)}{2}+\frac{\sigma_{m, i}(\theta) \varepsilon_{m}(\theta)}{2} \\
& +\tau_{m, i}(r, z) \varepsilon_{m}(r, z) r d z d r d \theta
\end{aligned}
$$

In (44) and (45), if $i=I$ then $x=0$ and $y=a$; and if $i=I I$ then $x=a$ and $y=L$.

By using stresses which are calculated in the previous section and substituting (7), (8), (13) and (14) into (44) and substituting (31) to (37) into (44), we find expressions for total strain energy in each region as below

$$
U_{I}=\eta_{1} a^{3}+\eta_{2} a^{2}+\eta_{3} a
$$

where $a$ is crack length and

$$
\begin{gathered}
\eta_{1}=\frac{2 \pi \tau_{s}^{2}}{3}\left(\frac{1}{r_{f} E_{f}}+\frac{V_{f}^{2}}{V_{m}^{2} E_{m}}\right) \\
\eta_{2}=\frac{-\pi r_{f} \sigma \tau_{s}}{V_{f} E_{f}}
\end{gathered}
$$

$$
\begin{aligned}
\eta_{3} & =\frac{\pi V_{f}^{2} \tau_{s}^{2}}{4 V_{m}^{2} G_{m} r_{f}^{2}} \\
& \times\left(4 r_{m}^{4} L n \frac{r_{m}}{r_{f}}-3 r_{m}^{2}+r_{f}^{4}-4 r_{f}^{2} r_{m}^{2}\right) \\
& +\frac{\pi r_{f}^{2}}{2}\left(\frac{\sigma^{2}}{E_{f}}+\frac{\tau_{s}^{2}}{2 G_{f}}\right)
\end{aligned}
$$


and

$$
U_{I I}=\mu_{1} a^{3}+\mu_{2} a^{2}+\mu_{3} a
$$

where

$$
\begin{gathered}
\mu_{1}=\frac{\pi}{3}\left(\frac{6 E \tau_{s}^{2}}{V_{m} E_{m} E_{f}}-\frac{2 \tau_{s}^{2}}{E_{f} r_{f}}-\frac{2 V_{f}^{2} \tau_{s}^{2}}{V_{m}^{2} E_{m}}\right) \\
\mu_{2}=\frac{\pi}{2}\left(\frac{2 V_{f}^{2} r_{f} \psi \phi\left(1+v_{m} \tau_{s}^{2}\right)}{V_{m}^{2} E_{m}}\right) \\
+\frac{\pi}{2}\left(\frac{6 E r_{f} \tau_{s}^{2}}{\psi V_{m} E_{m} E_{f}}-\frac{r_{f} \sigma \tau_{s}^{2}}{V_{f} E f}-\frac{2 E r_{f} \tau_{s}}{\psi V_{m} E_{m} E_{f}}\right) \\
\mu_{3}=\frac{\pi r_{f} \sigma \tau_{s}}{\psi V_{f}}\left(\frac{-V_{m}^{2} \psi_{2} \phi\left(1-v_{m}\right)}{4 V_{f} E}+\frac{3}{E_{f}}-\frac{4 r_{f}}{E_{f}}\right) \\
+\frac{-\pi r_{f}^{2} \sigma^{2}}{V_{f} E_{f}}\left(\frac{V_{m} E_{m}}{2 V_{f} E}+\frac{E_{f}}{E}-\frac{1}{V_{f}}+\frac{V_{f}}{2}\right)
\end{gathered}
$$

Also, for calculating $U_{W}$ we have

$$
U_{W}=U_{F}-U_{P}
$$

$U_{F}$ is the work done by friction stress $\tau_{s}$ and $U_{P}$ is the work done by tensile stress acting on fiber which are presented as ${ }^{[10]}$

$$
U_{F}=2 \pi r_{f} \int_{0}^{a} \tau_{s} v(z) d z
$$

where $v(z)$ is relative axial displacement between the fiber and the matrix which was given by Chiang ${ }^{[5]}$.

Then, by solving (51) we find

$$
U_{F}=\chi_{1} a^{3}+\chi_{2} a^{2}+\chi_{3} a
$$

where

$$
\begin{gathered}
\chi_{1}=\frac{-4 \pi}{3}\left(\frac{E \tau_{s}^{2}}{V_{m} E_{m} E_{f}}\right) \\
\chi_{2}=\pi\left(\frac{r_{f} \sigma \tau_{s}}{V_{f} E_{f}}-\frac{4 E r_{f} \tau_{s}}{\psi V_{m} E_{m} E_{f}}\right) \\
\chi_{3}=\pi\left(\frac{2 r_{f}^{2} \sigma \tau_{s}}{\psi V_{f} E_{f}}\right)
\end{gathered}
$$

And the work is done by friction stress $\tau_{s}$ is

$$
\begin{gathered}
U_{p}=\frac{\pi r_{f}^{2} \sigma}{V_{f}} v(0) \\
U_{p}=\xi_{1} a^{2}+\xi_{2} a+\xi_{3}
\end{gathered}
$$

where

$$
\begin{gathered}
\xi_{1}=\frac{\pi r_{f} \sigma \tau_{s}}{V_{f} E_{f}} \\
\xi_{2}=\left(\frac{-\sigma}{V_{f} E_{f}}+\frac{2 \tau_{s}}{\psi E_{f}}+\frac{\sigma}{E}\right) \frac{\pi r_{f}^{2}}{V_{f}} \sigma \\
\xi_{3}=\left(\frac{r_{f} V_{m} E_{m}+L \psi V_{f} E_{f}}{\psi V_{f}^{2} E_{f} E}\right) \pi r_{f}^{2} \sigma^{2}
\end{gathered}
$$

By Combining (42), (46), (50), (56) and (61), an expression for the energy release rate is obtained as

$$
U_{p}=\alpha_{1} a^{2}+\alpha_{2} a+\alpha_{3}
$$

where

$$
\alpha_{1}=\frac{E \tau_{s}^{2}}{r_{f} V_{m} E_{f} E_{m}}
$$

$$
\begin{aligned}
\alpha^{2}= & \frac{-V_{f}^{2} \psi \phi\left(1-v_{m}\right) \tau_{s}^{2}}{V_{m}^{2} E_{m}}+\frac{3 E \tau_{s}^{2}}{\psi V_{m} E_{m} E_{f}} \\
& -\left(\frac{\sigma}{2 V_{f} E_{f}}+\frac{E}{\psi V_{m} E_{m} E_{f}}\right) \tau_{s} \\
\alpha_{3} & =\frac{V_{m}^{2} \psi \phi\left(1+v_{m}\right) \sigma \tau_{s}}{8 V_{f}^{2} E}-\frac{3 \sigma \tau_{s}}{2 \psi V_{f} E_{f}} \\
& +\frac{r_{f} V_{m} E_{m} \sigma^{2}}{4 V_{f}^{2} E_{f} E}-\frac{r_{f}^{2} \tau_{s}^{2}}{8 G_{f}}-\frac{\pi V_{f}^{2} \tau_{s}^{2}}{8 V_{m}^{2} G_{m} r_{f}^{3}} \\
& \times\left(4 r_{m}^{4} \operatorname{Ln} \frac{r_{m}}{r_{f}}-3 r_{m}^{2}+r_{f}^{4}-4 r_{f}^{2} r_{m}^{2}\right)
\end{aligned}
$$

\section{Results}

We Show that the energy release rate is a second-order function of the cracked length $a$ when the material and geometry parameters are known. When an interfacial crack growth criterion $G \geq \Gamma_{i}$ is introduced, the critical crack length can be determined by

$$
\begin{gathered}
G \geq \Gamma_{i} \Rightarrow \alpha_{1} a^{2} \alpha_{2} a+\alpha_{3}-\Gamma_{i} \geq 0 \\
a_{c_{1,2}}=\frac{-\alpha_{2} \pm \sqrt{\alpha_{2}^{2}-4 \alpha_{1}\left(\alpha_{3}-\Gamma_{i}\right)}}{2 \alpha_{1}}
\end{gathered}
$$

Only the smaller $\alpha_{c}$ of the two roots of (70) is physically meaningful.

Fig. 2 shows Distributions of the energy release rate via the normalized cracked length which is extracted with an exact solution and good agreement between the methods is presented in this paper with the others.

As we can see in Fig. 2 the curves $G$ relative to normalized crack length have the first decreasing and then reincreasing tendency. The re-increasing part is physically meaningless because interfacial debonding appears only at $G \geq \Gamma_{i}$ and stops after the condition $G=\Gamma_{i}$ is satisfied. The effect of friction, between fiber and matrix in region I is considered in this paper as important factor for calculation of strain energy release rate that is illustrated in Fig. 3.

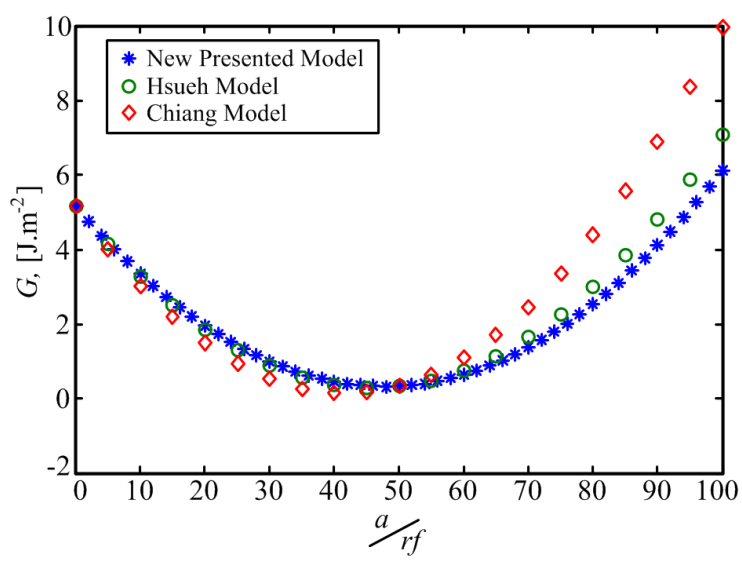

Fig. 2. Distributions of the energy release rate via the normalized cracked length 


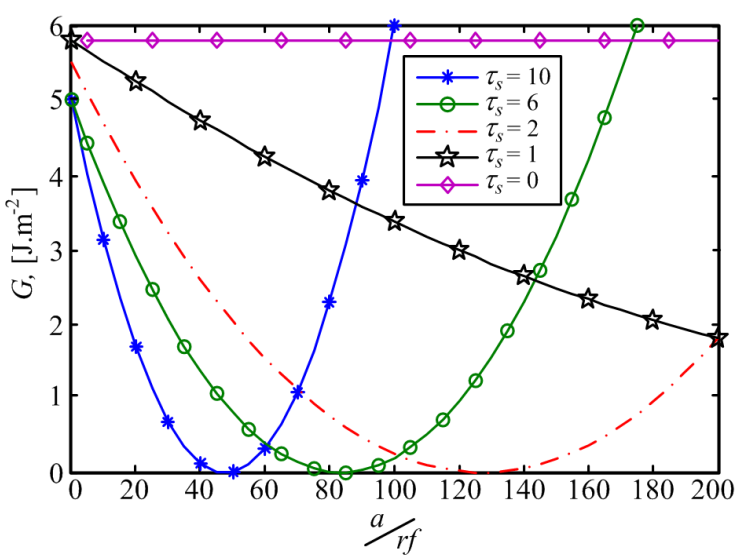

Fig. 3. The effect of friction between fiber and matrix in region II

According to Fig. 3, increasing friction stress $\tau_{s}$ results in a smaller $G$ at the same ${ }^{a /} r_{f}$, improving the interfacial debonding toughness $\Gamma_{i}$. The curves $G-\left({ }^{a} / r_{f}\right)$ tend to be smooth when the friction stress $\tau_{s}$ decreases and approaches the minimum value $\tau_{s}=0$, where the energy release rate reaches the maximum value $G=6.02\left[\frac{\mathrm{J}}{\mathrm{m}^{2}}\right]$ for the decreasing parts of curves.

\section{Conclusion}

For calculating strain energy release rate, we divided the single fiber embedded in a concentric cylindrical matrix into two regions. Region I is cracked and region II is intact region. Using stress equilibrium equations, boundary and continuity conditions for each region we calculate stresses separately and then according to minimum complementary energy principle, we define strain energy release rate as second order function of crack length.

The following conclusions are obtained

1. The presented method in this paper is feasible to determine critical debond length in fiber/matrix interfacial crack.

2. Numerical calculation results for fiber-reinforced composite, SiC/LAS, have good agreement with experimental data which obtained by other methods.

3 . The curves $G$ relative to normalized crack length have the first decreasing and then re-increasing tendency. The re-increasing part is physically meaningless because interfacial debonding appears only at $G>\Gamma_{i}$ and stops after the condition $G=\Gamma_{i}$ is sat- isfied.

4. When the interfacial friction between fiber and matrix at region I increase, the curves $G$ relative to the normalized crack length have sharp slope and when we have complete debonding in interface of fiber and matrix, $G$ is independent from crack length.

5. The shear effects in the fiber and matrix and Poisson's effect neglected by the shear-lag models become more remarkable with the increase of friction stress $\tau_{s}$ for suppressing the interface failure.

\section{REFERENCES}

[1] A. Hampe, G. Kalinka, and S. Meretz, "Advanced equipment for single-fiber pull-out test designed to monitor the fracture process," Composites, vol. 26, no. 1, pp. 40-46, 1995.

[2] K. Honda and Y. Kagawa, "Debonding criterion in the pushout process of fiber-reinforced ceramics," Acta materialia, vol. 44, no. 8, pp. 3267-3277, 1996

[3] C. H. Hsueh, "Crack-wake interfacial debonding criteria for fiber-reinforced ceramic composites," Acta materialia, vol. 44, no. 6, pp. 2211-2216, 1996.

[4] S. Ochiai, M. Hojo, and T. Inoue, "Shear-lag simulation of the progress of interfacial debonding in unidirectional composites," Composites science and technology, vol. 59, no. 1, pp. 77-88, 1999

[5] Y. C. Chiang, "On fiber debonding and matrix cracking in fiber-reinforced ceramics," Composites science and technology, vol. 61, no. 12, pp. 1743-1756, 2001.

[6] G. Rauchs and P. J. Withers, "Computational assessment of the influence of load ratio on fatigue crack growth in fibrereinforced metal matrix composites," International journal of fatigue, vol. 24, no. 12, pp. 1205-1211, 2002.

[7] G. Papakaliatakis and D. Karalekas, "Damage growth by debonding in a single fibre metal matrix composite: Elastoplasticity and strain energy density criterion," Theoretical and Applied Fracture Mechanics, vol. 53, no. 2, pp. 152157, 2010.

[8] V. I. Kushch, S. V. Shmegera, P. Brøndsted, and L. Mishnaevsky Jr., "Numerical simulation of progressive debonding in fiber reinforced composite under transverse loading," International Journal of Engineering Science, vol. 49, no. 1, pp. 17-29, 2011.

[9] A. C. Johnson, S. A. Hayes, and F. R. Jones, "The role of matrix cracks and fibre/matrix debonding on the stress transfer between fibre and matrix in a single fibre fragmentation test," Composites Part A: Applied Science and Manufacturing, vol. 43, no. 1, pp. 65-72, 2012.

[10] P. F. Liu, W. M. Tao, and Y. M. Guo, "Properties of frictional bridging in fiber pull-out for fiber-reinforced composites," Journal of Zhejiang University Science, vol. 6, no. 1, pp. 816, 2005.

[11] B. Budiansky and Y. L. Cui, "Toughening of ceramics by short aligned fibers," Mechanics of materials, vol. 21, no. 2, pp. 139-146, 1995. 